\title{
Loxoscelismo cutáneo visceral: actualización en el manejo a propósito de un caso
}

\author{
Isidora Harz-Fresno, Pilar Manterola, Magdalena Ruiz y Cristina Abud
}

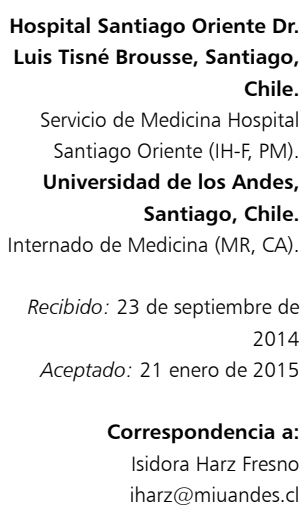

Hospital Santiago Oriente Dr. Luis Tisné Brousse, Santiago, Chile.

Servicio de Medicina Hospita Santiago Oriente (IH-F, PM) Universidad de los Andes, Santiago, Chile. Internado de Medicina (MR, CA).

Recibido: 23 de septiembre de 2014

Aceptado: 21 enero de 2015

Correspondencia a: Isidora Harz Fresno iharz@miuandes.cl

.

\section{Viscerocutaneous loxoscelism: case report and update on management}

Loxoscelism is a condition produced by the bite of Loxosceles laeta. It can present as cutaneous loxoscelism with only vascular dermal manifestations or as viscerocutaneus loxoscelism with systemic compromise and a mortality rate of 1 to $3 \%$. We report the case of an adult patient presenting viscerocutaneus loxoscelism, who was evolving with macrohematuria, edema, and progressive blisters, requiring treatment in the intensive care unit. He was treated according to the actual scientific evidence with antihistamines, corticosteroids, and dual antibiotic therapy covering Streptococcus spp., Staphylococcus spp., and anaerobes, particularly penicillin and tetracycline resistant $C$. perfringens. The use of dapsone and antiloxosceles-serum was avoided. The patient showed a favorable clinical evolution.

Key words: Loxosceles laeta, loxoscelism, viscerocutaneous loxoscelism.

Palabras clave: Loxosceles laeta, loxoscelismo, loxoscelismo cutáneo-visceral, loxoscelismo visceral.
\end{abstract}

\section{Introducción}

$\mathrm{E}$ 1 loxoscelismo es el cuadro clínico generado por el veneno de algunas especies de arañas del género Loxosceles. Existen tres especies sudamericanas causantes de loxoscelismo: Loxosceles laeta, Loxosceles intermedia y Loxosceles gaucho; la única presente en Chile es L. laeta ${ }^{1}$.

Las Loxosceles son de hábito nocturno, habitan preferentemente en espacios oscuros y secos, y se adaptan bien a ambientes domésticos ${ }^{2}$. La mayoría de las mordeduras ocurren de noche, al quedar la araña atrapada contra el cuerpo al dormir o al vestirse con ropa colgada por largo tiempo en armarios ${ }^{3}$. Las áreas principalmente afectadas son extremidades, tronco y pelvis, y finalmente la cara ${ }^{3}$.

El veneno es dermo-necrótico y víscero-tóxico; termolábil con propiedades necrosantes, hemolíticas, vasculíticas y coagulantes ${ }^{4}$. Posee numerosas enzimas, siendo la esfingomielinasa el componente más tóxico al desencadenar alteraciones que involucran el sistema del complemento y activación de neutrófilos ${ }^{1,5}$.

La forma de presentación más frecuente es el loxoscelismo cutáneo (LC) correspondiente a $83,3 \%$ de los casos $^{3}$. Se caracteriza por alteraciones vasculares graves en la piel, con áreas de vasoconstricción y otras de hemorragia, llevando rápidamente a isquemia local y a la constitución de una placa gangrenosa, de centro pálido rodeado por un área roja, edematosa y que evoluciona a azul-violácea, dura, estrellada y con centro umbilicado, denominada placa livedoide ${ }^{3,6}$. Se ha descrito además una variante únicamente edematosa de $\mathrm{LC}^{1}$.
El loxoscelismo cutáneo-visceral (LCV) es el cuadro clínico de mayor gravedad y se presenta en 16\% de los casos $^{3}$. Ocurre cuando el veneno alcanza la circulación sistémica, tanto por inoculación directa en un capilar, como por alteración de la permeabilidad, ejerciendo un gran poder hemolítico ${ }^{3}$. Ésta es la forma más grave de loxoscelismo, con una mortalidad de 1 a $3 \%{ }^{7}$. Se caracteriza por gran compromiso del estado general, acompañado de ictericia, palidez, hematuria, hemoglobinuria $y / o$ fiebre, durante las primeras 12 a $24 \mathrm{~h}$ desde la mordedura. La hemoglobinuria y hematuria están presentes en $100 \%$ de los LCV. La gravedad de la manifestación cutánea, no predice su evolución a un $\mathrm{LCV}^{7}$.

En Chile, el loxoscelismo es un problema de salud pública. El año 2005 el Centro de Información Toxicológica de la Universidad Católica de Chile (CITUC) atendió 2.831 llamadas telefónicas por sospecha de loxoscelismo, de los cuales 287 fueron confirmados ${ }^{3}$.

Las últimas guías clínicas chilenas para el manejo del loxoscelismo datan del año 2004. Las recomendaciones para el LC incluyen frío local, clorfenamina, antimicrobianos profilácticos (cloxacilina o flucloxacilina), analgésicos e inhibidores de polimorfonucleares (dapsona, colchicina) ${ }^{8}$. Frente a un cuadro de LCV se adiciona la monitorización estricta de la diuresis, el uso de bicarbonato en caso de hemoglobinuria, transfusiones de hemoderivados en caso de hemólisis grave y diálisis en caso de falla renal. Sugiere además, sin encontrarse su eficacia demostrada, el uso de corticoesteroides, de suero anti-loxosceles hasta seis horas luego de la exposición y de oxígeno hiperbárico ${ }^{8}$. 
La siguiente comunicación tiene por objetivo presentar la evolución de un caso de un adulto con LCV y difundir las recomendaciones actuales para el tratamiento del loxoscelismo, recalcando la importancia de la puesta al día en patologías frecuentes y potencialmente mortales de la epidemiología local.

\section{Caso clínico}

Paciente masculino de 27 años, con antecedentes de alcoholismo (un litro de ron diario) y policonsumo de drogas (cocaína, pasta base y cannabis). Consultó en el Servicio de Urgencia del Hospital Luis Tisné (HLT) por una lesión cutánea de 20 h de evolución, secundaria a una mordedura de araña en el tercio proximal del antebrazo derecho, en la cara ventral. Al examen presentaba una placa violácea central asociada a edema, eritema y dolor del antebrazo y de los dos tercios inferiores del brazo. Se evidenció una puerta de entrada de $4 \mathrm{~mm}$ con signos de necrosis en el antebrazo. A su ingreso el paciente se encontraba normotenso, taquicárdico, con saturación de oxígeno normal, eupneico y con temperatura axilar de $37,5^{\circ} \mathrm{C}$. Entre los exámenes de laboratorio del ingreso destacaba una leucocitosis, elevación de PCR e hiperbilirrubinemia de predominio indirecto con el resto de los exámenes en rangos de normalidad (Tabla 1).

Se inició aporte de volumen con solución fisiológica $0,9 \%$ a $120 \mathrm{cc} / \mathrm{h}$ i.v., y terapia con ceftriaxona $2 \mathrm{~g} /$ día i.v., metronidazol $500 \mathrm{mg} \mathrm{c} / 8 \mathrm{~h}$ i.v., hidrocortisona 100 $\mathrm{mg} \mathrm{c} / 8 \mathrm{~h}$ i.v., además de ketoprofeno $\mathrm{c} / 12 \mathrm{~h}$ i.v. y dipirona (metamizol) $1 \mathrm{~g} \mathrm{c} / 8 \mathrm{~h}$ i.v. Inicialmente evolucionó subfebril hasta $37,4^{\circ} \mathrm{C}$ y sin cambios de color en la orina.

A las 32 h de evolución, presentó hematuria macroscópica y un aumento del edema de la extremidad superior derecha, con la aparición de una flictena de aproximadamente $6 \mathrm{~mm}$ de diámetro en el sitio de mordedura y livideces en cara ventral del antebrazo (Figura 1A y B). Se mantuvo con aporte de volumen generoso e hidrocortisona i.v. Se inició clorfenamina $4 \mathrm{mg} \mathrm{c} / 8 \mathrm{~h}$ vo y se cambió ceftriaxona a cefazolina $1 \mathrm{~g} \mathrm{c} / 8 \mathrm{~h}$ i.v., manteniéndose con metronidazol. Se optimizó la analgesia con paracetamol $1 \mathrm{~g} \mathrm{c} / 8 \mathrm{~h}$ vo y tramadol $200 \mathrm{mg}$ i.v. en bomba de infusión continua. En los exámenes de laboratorio destacó un deterioro de la función renal y hepática y aumento de LDH (Tabla 2).

Por el compromiso renal se trasladó a Unidad de Tratamiento Intermedio (UTI) a las 44 h de evolución, destacando la aparición de múltiples flictenas en el antebrazo derecho (Figura 1C). En UTI evolucionó afebril, sin compromiso hemodinámico ni ventilatorio, con orinas más claras, persistiendo un leve tinte hemático. En la piel se observó una disminución del edema y calor local de la extremidad afectada con rotura de la flictena (Figura 1D). Los parámetros de laboratorio, a las $60 \mathrm{~h}$ de la mordedura, fueron normalizándose (Tabla 2). Al cuarto día de evolución, dada la mejoría clínica y de laboratorio, se trasladó a una sala de Medicina, desde donde el paciente se fugó del hospital, pese al inicio de la terapia de la

\begin{tabular}{|c|c|c|c|c|}
\hline \multicolumn{5}{|l|}{ Examen } \\
\hline Hematocrito & \multicolumn{2}{|l|}{$46 \%$} & Hemoglobina (g/dL) & 13,5 \\
\hline Leucocitos (K/UI) & \multicolumn{2}{|l|}{16.300} & Segmentados & $96,1 \%$ \\
\hline Plaquetas & \multicolumn{2}{|l|}{263.000} & PCR (mg/dL) & 5 \\
\hline Creatinina (mg/dL) & \multicolumn{2}{|l|}{0,97} & $\mathrm{Na}^{+} / \mathrm{K}^{+} / \mathrm{Cl}^{-}(\mathrm{mmol} / \mathrm{L})$ & $135 / 3,5 / 97$ \\
\hline CK total $(\mathrm{U} / \mathrm{L})$ & \multicolumn{2}{|l|}{128} & Lactato (mg/dL) & 13,6 \\
\hline TTPA (segundos) & \multicolumn{2}{|l|}{25,3} & Tiempo protrombina & $85 \%$ \\
\hline \multirow[t]{3}{*}{ Perfil hepático } & Bilirrubina total (mg/dL) & 3,44 & Bilirrubina indirecta (mg/dL) & 2,79 \\
\hline & GOT (U/L) & 55 & GPT (U/L) & 38 \\
\hline & GGT (U/L) & 42 & Fosfatasa alcalina (U/L) & 81 \\
\hline Sedimento de orina & \multicolumn{4}{|c|}{ Glóbulos blancos 0/campo, glóbulos rojos 0-2/campo, sin bacterias. } \\
\hline
\end{tabular}

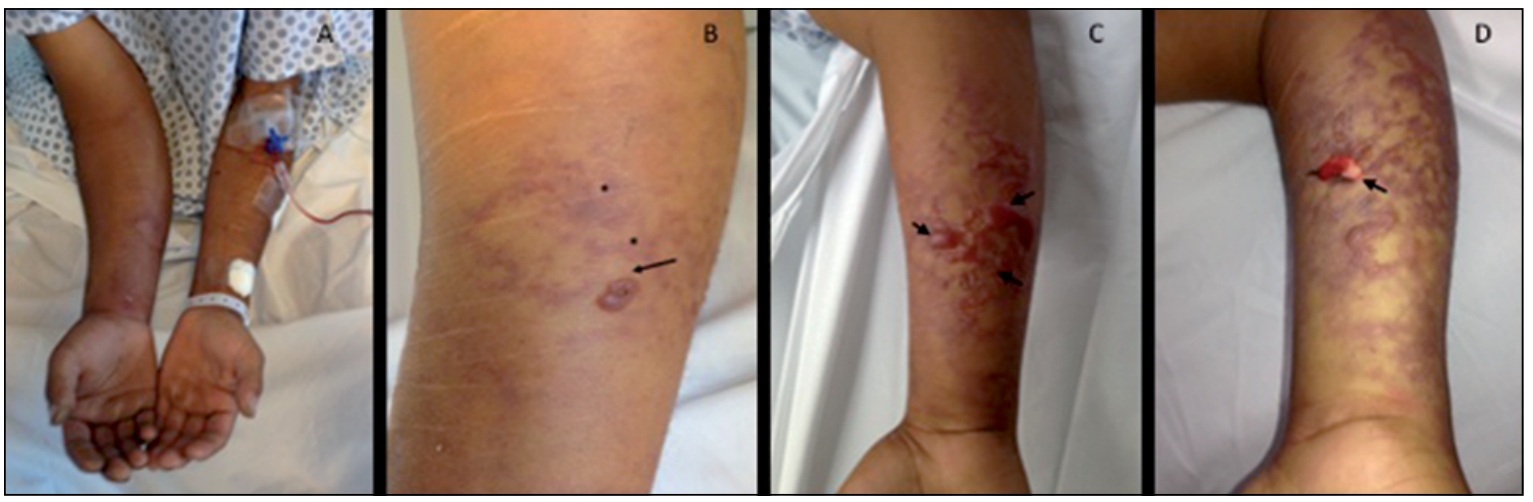

Rev Chilena Infectol 2015; 32 (2): 230-233
Figura 1. A) Edema de antebrazo derecho a las $32 \mathrm{~h}$ de evolución y B) flictena (flecha) y livideces $\left({ }^{*}\right)$. C) Múltiples flictenas (flechas) a las $44 \mathrm{~h}$ de evolución. D) Rotura de flictena a las $60 \mathrm{~h}$ de evolución (flecha). 


\begin{tabular}{|c|c|c|c|c|c|}
\hline $\begin{array}{l}\text { Evolución } \\
\text { Examen }\end{array}$ & $20 \mathrm{~h}$ (Ingreso) & & $32 \mathrm{~h}$ & $60 \mathrm{~h}$ & $84 \mathrm{~h}$ \\
\hline Creatinina (mg/dL) & 0,97 & & 1,37 & 1,03 & 0,9 \\
\hline CK total $(\mathrm{U} / \mathrm{L})$ & 128 & & 176 & & \\
\hline \multirow[t]{6}{*}{ Perfil hepático } & Bilirrubina total (mg/dL) & 3,44 & 4,33 & 2,77 & 1,49 \\
\hline & Bilirrubina indirecta $(\mathrm{mg} / \mathrm{dL})$ & 2,79 & & & \\
\hline & GOT (U/L) & 55 & 242 & 152 & 45 \\
\hline & GPT (U/L) & 38 & & & \\
\hline & GGT (U/L) & 42 & & & \\
\hline & Fosfatasa alcalina (U/L) & 81 & & & \\
\hline LDH (U/L) & & & 6.414 & 5.391 & 3.733 \\
\hline
\end{tabular}

poliadicción y alcoholismo con benzodiacepinas. Alcanzó a recibir cuatro días de tratamiento con antihistamínicos, corticoides y antibacterianos.

Al sexto día de la mordedura consultó nuevamente en el Servicio de Urgencia por dolor de la extremidad, destacando franca mejoría del edema, coloración violácea del antebrazo derecho, y flictenas en fase de cicatrización (Figura 2). El paciente nuevamente se dio a la fuga, sin volver a consultar en el HLT.

\section{Discusión}

El LCV es potencialmente mortal y requiere manejo oportuno. Actualmente la Guía Clínica del Ministerio de Salud de Chile (MINSAL) cumple 10 años, sin existir una actualización en el tratamiento del loxoscelismo y sin un claro manejo en casos de LCV. Sobre su recomendación acerca del uso de dapsona, sería más efectiva en la curación de la lesión en $\mathrm{LC}^{1}$; sin embargo, no está recomendada en $\mathrm{LCV}$, pues produce hemólisis con metahemoglobinemia, pudiendo confundirse y potenciar la hemólisis propia de $\mathrm{LCV}^{1,9}$, por lo que no se utilizó en el paciente. Sin embargo, sí se indicó clorfenamina, basado en la serie de casos de Schenone y cols. ${ }^{10}$, que asoció su uso a una reducción del dolor y edema, y a lo sugerido por el CITUC ${ }^{11}$.

Respecto al uso de corticoesteroides, la Guía Clínica del MINSAL lo sugiere, sin ser categórica al respecto ${ }^{8}$. La revisión sistemática de Manríquez y cols. ${ }^{1}$, hace referencia a la asociación entre su administración y un retardo en la cicatrización de la herida en los casos de LC, pero no menciona casos de LCV. Por otro lado, en el LCV los corticoesteroides sí podrían jugar un rol, dado su mecanismo inmunosupresor, así podría disminuir el efecto de la respuesta del sistema inmune que aumentaría el daño en el $\mathrm{LCV}^{12}$. Por lo anterior, y en vista de la rápida progresión del cuadro a LCV con un importante compromiso renal, se

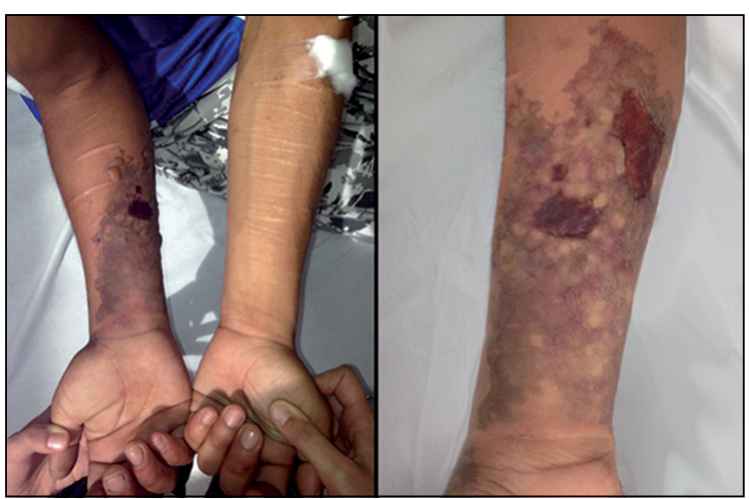

Figura 2. Sexto día de evolución: A) Mínimo edema. B) Coloración violácea del antebrazo derecho y flictenas en cicatrización.

decidió continuar con la administración de hidrocortisona i.v. en nuestro paciente.

En cuanto al suero anti-loxosceles existen estudios en animales que apoyan su efectividad ${ }^{13}$. Si bien es una intervención con gran potencial terapéutico, se ha asociado a efectos secundarios como reacciones alérgicas y no existe evidencia suficiente que apoye su uso en humanos ${ }^{1}$.

Otro punto importante es la terapia antibacteriana. $\mathrm{Su}$ objetivo es actuar en la lesión cutánea, siendo su uso profiláctico, para evitar infecciones secundarias por Streptococcus spp. y Staphylococcus spp. ${ }^{1}$, así como terapéutico dada la existencia de bacterias anaerobias como Clostridium spp. en la mandíbula y veneno de L. laeta ${ }^{14}$. El estudio realizado por Catalán y cols. ${ }^{14}$, demostró presencia de Clostridium perfringens resistentes a penicilina y tetraciclinas aisladas en ejemplares de L. laeta en Chile y que su presencia, asociada al veneno, aumenta la gravedad de los síntomas y la extensión de la dermonecrosis en conejos. Por lo anterior, en nuestro paciente se decidió asociar cefazolina, para cobertura de Streptococcus spp. y Staphylococcus spp., y metronidazol para cubrir bacterias anaerobias, especialmente $C$. perfringens.

\section{Conclusión}

A partir de la experiencia con nuestro paciente y de la potencial mortalidad del LCV, es que consideramos de carácter urgente la realización de trabajos de investigación de buena calidad que definan conductas con evidencia potente respecto al manejo del LCV, así como la actualización basada en la evidencia de la guía ministerial para su manejo.

\section{Resumen}

El loxoscelismo es producido por la mordedura de la araña Loxosceles laeta. Puede ser cutáneo, con alteracio- 
nes vasculares o cutáneo-visceral, con manifestaciones sistémicas y una mortalidad de 1 a $3 \%$. Se presenta el caso de un paciente adulto con loxoscelismo cutáneo-visceral, que evolucionó con hematuria macroscópica, edema y aparición de flictenas en forma progresiva en antebrazo, requiriendo ser trasladado a la Unidad de Tratamiento Intermedio. Fue manejado según la evidencia científica actual que recomienda el uso de antihistamínicos, corticoesteroides y terapia antibacteriana asociada para la cobertura de Streptococcus spp., Staphylococcus spp. y bacterias anaerobias, especialmente $C$. perfringens resistente a penicilina y tetracilina, evitando el uso de dapsona y de suero anti-loxosceles. Evolucionó de manera favorable.

\section{Referencias bibliográficas}

1.- Manríquez M J J, Silva V S. Loxoscelismo cutáneo y cutáneo-visceral: Revisión sistemática. Rev Chil Infectol 2009; 26: 420-32.

2.- Isbister G K, Fan H W. Spider bite. Lancet 2001; 378: 2039-47.

3.- Ríos J C, Pérez M, Sánchez P, Bettini M, Mieres J J, Paris E. Prevalence and epidemiology of Loxosceles laeta bite. Analysis of consultations to a poison control center. Rev Med Chile 2007; 135: 1160-5.

4.- Ministerio de Salud de Chile. Guía clínica para el manejo y tratamiento de mordedura de la araña del rincón (Loxosceles laeta). Santiago de Chile, 2004. Disponible en http:// cituc.uc.cl/publicaciones/7-guía-clínica-para- manejo-de-mordedura-de -araña-de-rincón. (Fecha de acceso: 17 de marzo de 2015).

5.- Swanson D L, Vetter R S. Loxoscelism. Clin Dermatol 2006; 24 (3): 213-21.

6.- Instituto de Salud Pública de Chile. Araña de los rincones. Disponible en: http:// www.ispch.cl/oirs/SIAC_2007/siac. php?page $=$ A\&respuesta $=$ ARA $\% D 1 A \% 20$ DE\%20LOS\%20RINCONES\&cod=9. (Fecha de acceso: 17 de marzo de 2015).

7.- Ríos J C, Paris E, Apt W, Ristori L, Ramírez G. Guía clínica para el manejo de la mordedura de araña de rincón. Loxosceles laeta. Agosto, 2004.

8.- Glied M, Rico M J. Treatment of autoimmune blistering diseases. Dermatol Clin 1999; 17: 431-40.

9.- $\quad$ Schenone H. Estudio de 27 casos de Loxoscelismo. Bol Chil Parasitol 1959; 14: 7-13.
10.- Guía Clínica para el manejo de la mordedura de araña de rincón. Loxosceles laeta. Centro de Información Toxicológica Pontificia Universidad Católica de Chile ( CITUC). Disponible en: www.cituc.cl/LoxoscelesLaeta. pdf. (Fecha de acceso: 17 de marzo de 2015).

11.- Hogan C J, Barbaro K C, Winkel K. Loxoscelism: old obstacles, new directions. Ann Emerg Med 2004; 44: 608-24.

12.- Pauli I, Puka J, Gubert I C, Minozzo J C. The efficacy of antivenom in loxoscelism treatment. Toxicon 2006; 48: 123-37.

13.- Catalán A, Espoz M C, Cortés W, Sagua H, González J, Araya J E. Tetracycline and penicillin resistant Clostridium perfringens isolated from the fangs and venom glands of Loxosceles laeta: its implications in loxoscelism treatment. Toxicon 2010; 56: 890-6. 